\title{
Special issue - Towards a better understanding of Botryosphaeriales
}

\author{
AJL Phillips \\ University of Lisbon, Faculty of Sciences, Biosystems and Integrative Sciences Institute (BioISI), Campo Grande, 1749- \\ 016 Lisbon, Portugal \\ E-mail: alan.jl.phillips@gmail.com
}

Phillips AJL 2017 - Special issue - Towards a better understanding of Botryosphaeriales. Mycosphere 8(2), 102, Doi 10.5943/mycosphere/8/2/1

This editorial will be updated once all papers are uploaded Authors are invited to submit papers to this special issue through Mycosphere 